\title{
Comunicação
}

[Communication]

\section{Ocorrência de Hepatozoon spp. (Apicomplexa, Hepatozoidae) em serpentes do gênero Bothrops de cativeiro}

\author{
[Occurrence of Hepatozoon spp. (Apicomplexa, Hepatozoidae) in snakes \\ of genus Bothrops in captivity]
}

\author{
V. Glaser, A.P. Boni, C.A.C. Albuquerque*
}

Departamento de Ciências Naturais - FURB

Rua Antônio da Veiga, 140

89012-500 - Blumenau, SC

O gênero Hepatozoon é o grupo de protozoários intracelulares mais comuns encontrados em serpentes (Telford, 1984; Wozniak et al., 1994; Smith, 1996), e seu ciclo biológico requer dois hospedeiros: um invertebrado para a reprodução sexuada e outro vertebrado para a assexuada (Biasi et al., 1989). Os gametócitos desses protozoários, nos répteis, são observados parasitando os eritrócitos (Smith, 1996).

$\mathrm{Na}$ literatura, para o sul do país, não está documentada a presença desse parasito em serpentes mantidas em cativeiro. Dessa forma, esta pesquisa foi realizada com o objetivo de verificar a presença de parasitos do gênero Hepatozoon nas amostras de sangue de Bothrops jararaca $(\mathrm{n}=10)$ e Bothrops jararacussu $(\mathrm{n}=10)$ mantidas no serpentário da Universidade Regional de Blumenau e nos Zoológicos dos municípios de Brusque e Pomerode, SC. A coleta do sangue foi feita por punção da veia caudal (Kaplan, 1968), e o esfregaço sangüíneo confeccionado imediatamente. As lâminas foram coradas com May-Grünwald e Giemsa e analisadas utilizando micrômetro ocular na objetiva de imersão (aumento 1000x), e microscópio Olympus CX31. O parasitismo nos eritrócitos foi determinado mediante contagem de 10.000 células (O’Dwyer et al., 2004), detectando a presença de gametócitos de Hepatozoon spp. Foi realizada morfometria de eritrócitos infectados $(\mathrm{n}=50)$ e não infectados $(\mathrm{n}=50)$, assim como dos parasitos.

Das serpentes analisadas, 50\% apresentaram eritrócitos infectados com Hepatozoon sp., sendo seis em serpentes $(60 \%)$ da espécie $B$. jararaca e quatro $(40 \%)$ da $B$. jararacussu. O'Dwyer et al. (2003) verificaram prevalência de $16,4 \%$, em várias espécies de serpentes estudadas. No mesmo trabalho, na espécie $B$. jararaca, foi encontrada prevalência de $35,3 \%$ de serpentes infectadas, percentual menor que o observado no presente estudo para a mesma espécie.

Poucas células apresentaram o parasito, não representando $1 \%$ dos eritrócitos em $90 \%$ das serpentes infectadas. $O$ baixo nível de parasitemia também foi encontrado por Santos et al. (2005), com variação sazonal de Hepatozoon spp. em eritrócitos de Boa constrictor amarali e Hydrodynastes gigas. Biasi et al. (1972) observaram que este protozoário pode estar presente no hospedeiro sob forma latente ou com baixo nível de parasitemia.

Sugere-se que a infecção verificada por Hepatozoon spp. pode ter ocorrido no ambiente natural ou no cativeiro. Neste caso, pode ser atribuída à presença de carrapatos ou, ainda, à alimentação dos animais. Segundo O'Dwyer et al. (2003), a infecção pode ocorrer pela ingestão

Recebido em 22 de fevereiro de 2008

Aceito em 2 de setembro de 2008

* Autor para correspondência (corresponding author)

E-mail: claudia@furb.br 
de um hospedeiro vertebrado intermediário infectado ou por outros artrópodes.

Os gametócitos foram encontrados freqüentemente deslocando o núcleo dos eritrócitos, aspecto já observado por Pessôa e Biasi (1973), Silva (1975) e Jakes et al. (2003). Não foram observadas diferenças no tamanho dos eritrócitos que continham o parasito, cujas dimensões foram de $13,74 \mu \mathrm{m}( \pm 1,12) \times 4,5 \mu \mathrm{m}$ $( \pm 0,65)$, maior que o tamanho observado em Hepatozoon caimani (Lainson et al., 2003) e de comprimento menor que o descrito para Hepatozoon sauritus (Telford Jr. et al., 2004; Telford Jr. et al., 2005a), Hepatozoon boigae (Jakes et al., 2003), Hepatozoon priapus e
Hepatozoon confusus (Telford Jr. et al., 2005a). O tamanho do parasito intraeritrocitário foi semelhante ao da espécie Hepatozoon polytopis (Telford Jr. et al., 2005a;b). Todos os gametócitos das espécies citadas assemelham-se morfologicamente, diferenciando-se principalmente em relação ao tamanho e à posição do núcleo.

A prevalência desse parasito é importante para o melhor conhecimento da saúde dos animais que chegam ao cativeiro, pois são escassos os dados a respeito dessa parasitose.

Palavras-chave: serpente, Bothrops, Hepatozoon spp., parasitismo

\section{ABSTRACT}

The occurrence of Hepatozoon gamont in the blood cells of Bothrops jararaca and B. jararacussu in captivity was analyzed. The prevalence of infection by Hepatozoon spp. was $50 \%$ and few erythrocytes contained the gamonts. Results suggest that the infection by Hepatozoon spp. occurred in the natural environment or after the captivity.

Keywords: snake, Bothrops, Hepatozoon spp., parasitism

\section{REFERÊNCIAS BIBLIOGRÁFICAS}

BIASI, P.; PESSOA, S.P.; VIEIRA, S.C.G. Nota sobre longa latência de infecção por hemogregarina em uma serpente peçonhenta: Bothrops moojeni Hoge, 1965. Atas Soc. Biol., v.15, p.71-73, 1972.

BIASI, P.; CARDOSO JUNIOR, R.B.; SANTOS, S.M.A. Presença de Hepatozoon plimmeri (Sambom, 1909) - Coccidia, Haemogregarinidae - em exemplar de Bothrops jararaca (Wied, 1824) - Serpentes, Viperidae, Crotalinae - mantido em cativeiro. Mem. Inst. Butantan, v.51, p.117-121, 1989.

JAKES, K.A.; O'DONOGHUE, P.J.; WHITTIER, J. Ultrastructure of Hepatozoon boigae (Mackerras, 1961) nov. comb. from brown tree snakes, Boiga irregularis, from northern Australia. Parasitol. Res., v.90, p.225$231,2003$.

KAPLAN, H. Techniques of collection in ectothermic of body fluids, excreta and vital products for laboratory examination. Lab. Anim. Care, v.18, p.131-142, 1968.
LAINSON, R.; PAPERNA, I.; NAIFF, R.D. Development of Hepatozoon caimani (Carini, 1909) Pessôa, De Biasi \& De Souza, 1972 in the caiman Caiman c. crocodilus, the frog Rana catesbeiana and the mosquito Culex fatigans. Mem. Inst. Oswaldo Cruz, v.98, p.103-113, 2003.

O’DWYER, L.H.; MOÇO, T.C.; SILVA, R.J. Description of the gamonts of a small species of Hepatozoon spp.(Apicomplexa, Hepatozoidae) found in Crotalus durissus terrificus (Serpentes, Viperidae). Parasitol. Res., v.92, p.110-112, 2004.

O’DWYER, L.H.; MOÇO, T.C.; BARELLA, T.H. et al. Prevalence of Hepatozoon spp. (Apicomplexa, Hepatozoidae) among recently captured Brazilian snakes. Arq. Bras. Med. Vet. Zootec., v. 55, p.309-314, 2003.

PESSÔA, S.B.; BIASI, P. Nota taxonômica sobre cistos esporogônicos de algumas espécies de Hepatozoon (Sporozoa, Haemogregarinidae) parasitas de serpentes brasileiras. Mem. Inst. Butantan, v.37, p.299-307, 1973.

SANTOS, M.M.V.; O’DWYER, L.H.; SILVA, R.J. Seasonal variation of Hepatozoon spp. (Apicomplexa, Hepatozoidae) parasitemia from 
Boa constrictor amarali (Serpentes, Boidae) and Hydrodynastes gigas (Serpentes, Colubridae). Parasitol. Res., v.97, p.94-97, 2005.

SILVA, E.O.R. Ciclo evolutivo do Hepatozoon triatomae (Sporozoa, Haemogregarinidae) parasita de triatomíneos. Rev. Saude Publ., v.9, p.383-391, 1975.

SMITH, T.G. The genus Hepatozoon (Apicomplexa: Adeleina). J. Parasitol., v.82, p.565-585, 1996.

TELFORD JR., S.R. Reptilian hemoparasites. In: HOFF, G.L.; FRYE, F.L; JACOBSON, E.R. (Eds.). Diseases of amphibians and reptiles. New York: Plenum Publishing, 1984. p.385-517.

TELFORD JR., S.R.; ERNST, J.A.; CLARK, A.M. et al. Hepatozoon sauritus: a polytopic hemogregarine of three genera and four species of snakes in north Florida, with specific identity verified from genome analysis. J. Parasitol., v.90, p.352-358, 2004.

TELFORD JR., S.R.; BUTLER, J.F.; MOLER, P.E. Two additional Hepatozoon species (Apicomplexa: Hepatozoidae) from the southern black racer, Coluber constrictor priapus (Serpentes: Colubridae), in northern Florida. J. Parasitol., v.91, p.139-143, 2005a.

TELFORD Jr., S.R.; BUTLER, J.F.; TELFORD, R.S. Hepatozoon polytopis n. sp. parasitic in two genera and species of colubrid snakes in southern Florida. J. Parasitol., v. 91, p.144-147, 2005 b.

WOZNIAK, E.J.; TELFORD Jr., S.R., MCLAUGHLIN, G.L. Employment of the polimerase chain reaction in the molecular differentiation of reptilian hemogregarines and its application to preventative zoological medicine. J. Zoo. Wildl. Med., v.23, p.538-547, 1994. 